\title{
Determining Weights By Entropy Measures In Case Of Heteroscedasticity
}

\author{
Hatice Çiğdem Çin, Atıf EVREN
}

\begin{abstract}
In simple regression analysis, the dependent variable is assumed to have constant variance at different levels of independent variable. Whenever the assumption of constant variance fails, some remedies like the weighted least squares, or Box-Cox transformation may be helpful. While Box-Cox approach is based on a nonlinear transformation on the dependent variable, in the weighted least squares methodology, independent variable is rescaled by weights to maintain constant variance. Although there is already a large literature on this issue, determining the weights seems a major problem. In this study, the weights are alternatively calculated by entropy approach, since statistical entropy, and variance conveys similar information about a probability distribution. In this study, by exploiting the normality assumption of linear models, the weights are determined by the reciprocals of Shannon, Tsallis and Renyi entropies of normal distribution. The weighting procedure has been applied on some simulated data having nonconstant variance. In some applications we have shown that weighting by Tsallis and/or Rényi entropies produced better goodness of fit results in terms of coefficient of determination, and the mean square error.
\end{abstract}

Index Terms - Heteroscedasticity, entropy, weighted least squares, Shannon entropy, Tsallis entropy, Rényi entropy.

\section{INTRODUCTION}

One of the main assumptions of linear regression model is homoscedasticity. In other words, the variances of dependent variable at each level of independent variable/variables are the same. Heteroscedasticity, or nonconstancy of variances can be detected alternatively. The simplest way is to use visual representation of data, although relying only on visual means may be misleading. Therefore some statistical tools like White Test, or Park's Test are used. Once the heteroscedasticity is detected, to increase the goodness of fit, a kind of weighing procedure is followed. One way is to give more weights to the levels of independent variable/variables whose variabilities are lower. The Box-Cox transformation can also be used to avoid heteroscedasticity. Alternatively, although it's not in common usage, weighting with the reciprocal of entropy can be used. Shannon, Renyi and Tsallis are different types of entropy, and all of them could be used for weighing the data. Indeed, Rényi and Tsallis formulations of entropy bring more flexibility than Shannon entropy, due to their parametric nature.

Hatice Ciğdem Cin, Yildiz Technical University, Faculty of Sciences and Literature , Department of Statistics Davutpasa, Esenler, 34210, Istanbul,Turkey

Attf EVREN, Yildiz Technical University, Faculty of Sciences and Literature, Department of Statistics Davutpasa, Esenler, 34210, Istanbul, Turkey

\section{REGRESSION ANALYSIS}

The simplest linear model involves only one independent variable, and a constant. The change on the mean of dependent variable is related to only one independent variable. The true mean of $Y_{\mathrm{i}}$ is denoted by $E\left(Y_{\mathrm{i}}\right)$. It is stated as below:

$$
E\left(Y_{i}\right)=\beta_{0}+\beta_{1} X_{i}
$$

Here, $\beta_{0}$ is the intercept, and $\beta_{1}$ is the slope. The real $Y_{i}$ can be found by adding an error item to the true mean of $Y_{i}$ as

$$
Y_{i}=\beta_{0}+\beta_{1} X_{i}+e_{i}
$$

The index $i$ indicates the particular observational unit, $i=1,2 \ldots \ldots n \cdot X_{i}{ }^{\prime} s$ are observed without any measurement error. The $Y_{\mathrm{i}}$ and $X_{\mathrm{i}}$ are paired observations; both are measured on every observational unit. The random errors $e_{i}$ 's are assumed to distribute normally with zero mean, and a common variance $\sigma^{2}$, and to be pairwise independent.

These assumptions imply that the $Y_{i}$ 's also have common variance $\sigma^{2}$, and are pairwise independent, since the only random element in the model is $e_{\mathrm{i}}$. For inferential purposes, the random errors are assumed to be normally distributed, which implies that the $Y_{\mathfrak{i}}$ 's are also normally distributed.

\section{LEAST SQUARES ESTIMATION}

Let $\hat{\beta}_{0}$ and $\hat{\beta}_{1}$ be numerical estimates of the parameters $\beta_{0}$ and $\beta_{1}$, respectively. The least squares principle chooses $\hat{\beta}_{0}$ and $\hat{\beta}_{1}$ that minimize the sum of squares of the residuals,

$$
\mathrm{SS}(\operatorname{Res})=\sum_{\mathrm{i}=1}^{\mathrm{n}}\left(\mathrm{Y}_{\mathrm{i}}-\widehat{\mathrm{Y}}_{\mathrm{i}}\right)^{2}=\sum e_{\mathrm{i}}^{2}
$$

where $\widehat{Y_{1}}=\widehat{\beta_{0}}+\widehat{\beta_{1}} X_{\mathrm{i}}$. In matrix notation, we can find the vector $\beta$ which is $2 \times 1$ matrix, and includes the estimates of $\beta_{1}$ and $\beta_{2}$. It is as stated below:

$$
\hat{\beta}=\left(X^{\prime} X\right)^{-1}\left(X^{\prime} Y\right)
$$

where

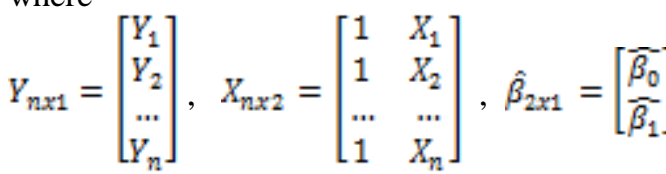

The variances of parameters are given by

$$
\operatorname{Var}(\beta)=\left(X^{\prime} X\right)^{-1} \sigma^{2}
$$

Two general goodness of fit statistics for linear regression models are $R^{2}$, and mean squares error (MSE). $R^{2}$ or coefficient of determination is defined as

$$
R^{2}=1-\frac{\sum_{i=1}^{n} \theta_{i}^{2}}{\sum_{i=1}^{n}\left(y_{i}-\eta\right)^{2}}
$$

Verbally, $R^{2}$ measures the proportion or percentage of the total variation in $\mathrm{Y}$ explained by the regression model (Rawlings, 1998). Adjusted $R^{2}$ is defined similarly as

$$
R_{a d j}^{2}=1-\frac{(n-1)}{(n-p)} \frac{\sum_{i=1}^{n} e_{i}^{2}}{\sum_{i=1}^{n}\left(v_{i}-q\right)^{2}}
$$

Finally, the mean squares error MSE is defined as

MSE $=\frac{S S[\mathrm{ReS}]}{n-p}$

where $p$ is the number of parameters of regression function. 


\section{White's and Park's Tests for Heteroscedasticity}

The general test of heteroscedasticity proposed by White does not rely on the normality assumption, and is easy to implement.White test can be a test of (pure) heteroscedasticity, or specification error, or both. It has been argued that if cross-product terms are present, then it is a test of both heteroscedasticity, and specification bias.(Gujarati, 2004).

Park formalizes the graphical method by suggesting that $\sigma_{i}^{2}$ is some function of the explanatory variable $X_{\mathrm{f}}$. If this relation is found to be statistically significant, it would suggest that heteroscedasticity is present in the data. One may use the Park test as a strictly explanatory method (Gujarati, 2004).

\section{Weighted Least Squares}

Let the weights $w_{i}$ be contained in the diagonal matrix of weights

$$
\left.W_{n x m}=\left[\begin{array}{ccc}
w_{1} & \cdots & 0 \\
\vdots & \cdots & \vdots \\
0 & \cdots & w_{n}
\end{array}\right]\right]
$$

The weighted least squares estimators of the regression coefficients are

$$
\hat{\beta}_{W}=\left(X^{t} W X\right)^{-1} X^{v} W Y
$$

When the variances are not equal, the weights are chosen to be inversely proportional to variances (Neter et all, 1985). The estimated variance-covariance matrix is

$S_{p x p}^{2}=M S E_{W}\left(X^{t} W X\right)^{-1}$

where

$M S E_{W}=\frac{\sum w_{i} e_{i}^{2}}{n-p}$

\section{Box-Cox Transformation}

Box-Cox transformation aims to convert a set of observations into a set of observations from a normal distribution with constant variance. The transformation involves a parameter estimated from the data using maximum likelihood (Upton\&Cook, 2006)). For each real number $\lambda_{x}$ and positive number $\mathrm{Y}$, the Box-Cox transformation is given by

$$
Y_{i}^{(\lambda)}= \begin{cases}\frac{Y_{i}^{\lambda}-1}{\lambda} & \text { for } \lambda \neq 0 \\ \ln \left(Y_{i}\right) & \text { for } \lambda=0\end{cases}
$$

In other words, the regression models with the same explanatory variables, but with different values of $\lambda$ are run to get the best goodness of fit statistics.

\section{Entropy}

Entropy is an indicator of reversibility; when there is no change of entropy, the process is reversible. An increase in entropy is a decrease of available energy. In general, an increase in entropy means decrease in order. Disorder in the sense of unpredictability based on a lack of knowledge of the positions and velocities of molecules ( Pierce,1980).

The entropy of a statistical experiment, is a measure of uncertainty (Khinchin, 1957). So in a statistical sense, entropy and the amount of information are two closely related concepts. Uncertainty is not present after a statistical experiment is conducted. Therefore entropy is the amount of information that can be provided by sampling. For some concepts and applications of statistical entropy, one can also refer to Cover\&Thomas (2006), Ash (1990), Reza(1994), Kullback(1996), and Rényi (2007).

\section{Shannon Entropy}

Let the discrete random variable $X$ takes on the value $x_{1}, x_{2}, \ldots, x_{k}$ with respective probabilities $p_{1}, p_{2}, \ldots, p_{k}$ Shannon entropy is defined as

$$
H_{s}=-\sum_{i=1}^{k} p_{i} \log p_{i}
$$

Let $\hat{H}$ is the estimator of Shannon entropy. It is calculated as

$$
\widehat{H}_{g}=-\sum_{i=1}^{k} \hat{p}_{i} \log \hat{p}_{i}
$$

Here $\hat{p}_{i}$ probabilities are estimated by maximum iikelihood method. Although this estimator is biased, ncreasing the sample size can reduce the amount of bias. The variance of Shannon entropy is found as (Zhang Xing, 2013).

$$
V(\hat{H}) \cong \frac{1}{n}\left(\sum_{i=1}^{K} p_{i} l^{2} p_{i}-H^{2}\right)+\frac{K-1}{2 n^{2}}
$$

\section{Rényi Entropy}

Rényi entropy is defined as

$H_{R}=\frac{\log \sum_{i=1}^{K} p_{i}^{a}}{1-a}$ for $\quad a>0, \quad$ and $\alpha \neq 1$

Rényi entropy is also called as type of entropy (Ullah, A. 1996). As the parameter approaches unity, Rényi entropy approaches to Shannon entropy. Thus Shannon entropy is a special case of Rényi entropy. The variance of Rényi entropy is given as follows (Pardo, 2006);

$$
V\left(\hat{H}_{R}\right) \cong \frac{1}{n}\left[\left(\frac{a}{a-1}\right)^{2}\left(\sum_{i=1}^{K} p_{i}^{2 a-1}-\left(\sum_{i=1}^{K} p_{i}^{a}\right)^{2}\right)\right]
$$

\section{Tsallis Entropy}

Tsallis (or Havrda-Charvat) entropy is known as

$$
H_{T}=\frac{1-\sum_{i=1}^{K} p_{i}^{a}}{a-1} \text { for } a>0 \text { and } a \neq 1
$$

The variance of this entropy estimator is given by

$$
V\left(H_{T}\right) \cong \frac{1}{n}\left[\left(\frac{a}{a-1}\right)^{2}\left(\sum_{i=1}^{K} p_{i}^{2 a-1}-\left(\sum_{i=1}^{K} p_{i}^{a}\right)^{2}\right)\right] \text { (20) }
$$

The formulas of Shannon, Renyi and Tsallis entropy for normal distribution can be calculated as below:

\begin{tabular}{|l|l|}
\hline & Normal Distribution \\
\hline Shannon entropy & $\ln (\sigma \sqrt{2 \pi \theta})$ \\
\hline Renyi entropy & $\ln (\sigma \sqrt{2 \pi \theta})-\frac{\ln \alpha}{2(1-\alpha)}$ \\
\hline Tsallis entropy & $\frac{\sqrt{\alpha}-(\sigma \sqrt{2 \pi})^{1-\alpha}}{\sqrt{\alpha}(\alpha-1)}$ \\
\hline
\end{tabular}

Table1: Entropies for normal distribution

\section{APPLICATION}

We have generated and used some artificial heteroscedastic data given in appendix data-1, data- 2 , data-3 and data- $4 \mathrm{We}$ then fitted linear regression models, each having its own weighting methodology. Then we compared the goodness of fit statistics of each linear regression model, after weighting, and performing the weighted least squares technique. Note that initially (as a classical approach) we took the reciprocals of the sample standard deviations calculated at each X-level as the weights. Then we repeated the same procedure by taking the weights as the reciprocals of entropies of normal distribution calculated at the X-levels. Note that since Tsallis, and Rényi entropies have parametric nature, we performed various weighting techniques by giving different values to the $\alpha$ parameter. Finally, as an alternative to the weighted least squares we adopted Box-Cox approach.The three goodness of fit statistics used are $R^{2}, R_{a d j}^{2}$ and $M S E$.

From Figure 1, and Figure 2, the heteroscedastic nature of data is obvious. These data were produced by adding random 
numbers whose variances are increasing systematically according to the levels of independent variables. In other words, the bigger the independent variable, the bigger the variance of simulated values. Note that random numbers are generated from normal distributions with different variances. Note too that for the first data set, there are 5 dependent observations for each level of independent variable, and for the second, third, and fourth data sets there are 25 dependent observations generated. By checking regression results summarized in Tables 2-4, we may say that weighing has a positive effect on increasing $R^{2}, R_{a d j}^{2}$ and/or decreasing MSE . Besides we have observed that in many cases using the reciprocals of Tsallis, and Rényi entropies as the weights, produced much better goodness of fit statistics.

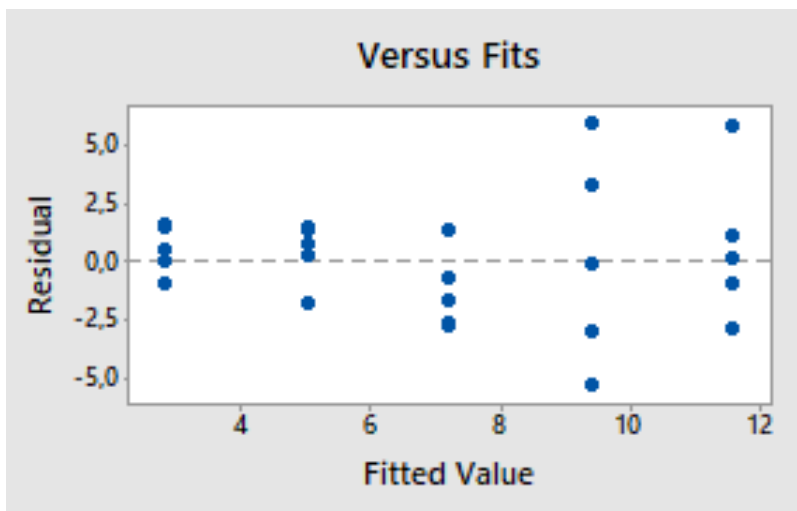

Fig 1: The scatter diagram of data-1

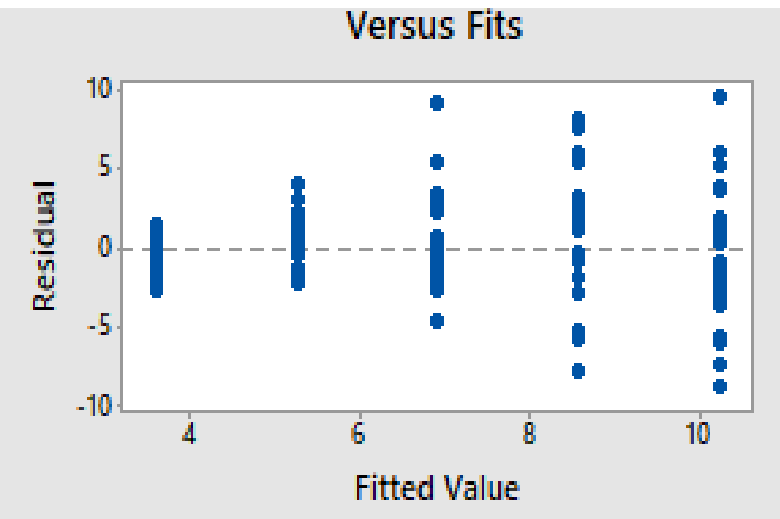

Fig 2: The scatter diagram of data-2

\begin{tabular}{l|r|r|r|} 
Data-1 & \multicolumn{1}{|l|}{$\mathrm{R}^{2}$} & \multicolumn{1}{l|}{$\mathrm{R}^{2} \mathrm{adj}$} & \multicolumn{1}{l|}{ MSE } \\
\hline Classical & $59.3 \%$ & $57.53 \%$ & 7.173 \\
\hline Weighted with OLS & $65.84 \%$ & $64.35 \%$ & 1.052 \\
\hline Shannon & $63.28 \%$ & $61.68 \%$ & 2.881 \\
\hline Renyi $\alpha=0,5$ & $62.4 \%$ & $60.77 \%$ & 2.241 \\
\hline Renyi $\alpha=1,5$ & $62.71 \%$ & $61.09 \%$ & 2.467 \\
\hline Box-cox & $63.24 \%$ & $61.65 \%$ & 0.094 \\
\hline Tsallis $\alpha=1,5$ & $61.23 \%$ & $59.55 \%$ & 4.064 \\
\hline Tsallis $\alpha=0,5$ & $63.96 \%$ & $62.39 \%$ & 0.027 \\
\hline Tsallis $\alpha=1 / 9$ & $66.27 \%$ & $64.81 \%$ & 0.021 \\
\hline Tsallis $\alpha=1 / 10$ & $66.22 \%$ & $64.75 \%$ & 0.068 \\
\hline Renyi $\alpha=1 / 9$ & $60.84 \%$ & $59.14 \%$ & 1.113 \\
\hline
\end{tabular}

Table I: Goodness of fit statistics model for data-1

\begin{tabular}{|c|c|c|c|}
\hline Data-2 & $R^{2}$ & $R^{2}$ adj & MSE \\
\hline Classical & $35.24 \%$ & $34.71 \%$ & 10.260 \\
\hline Weighted with OLS & $44.63 \%$ & $44.18 \%$ & 1.589 \\
\hline Shannon & $39.06 \%$ & $38.57 \%$ & 4.086 \\
\hline Renyi $\alpha=0,5$ & $38.25 \%$ & $37.75 \%$ & 3.175 \\
\hline Renyi $\alpha=1,5$ & $38.54 \%$ & $38.04 \%$ & 3.497 \\
\hline Box-cox & $35.39 \%$ & $34.87 \%$ & 0.371 \\
\hline Tsallis $\alpha=1,5$ & $36.88 \%$ & $36.37 \%$ & 5.810 \\
\hline Tsallis $\alpha=0,5$ & $39.38 \%$ & $38.89 \%$ & 0.648 \\
\hline Tsallis $\alpha=1 / 9$ & $44.24 \%$ & $43.79 \%$ & 0.032 \\
\hline Tsallis $\alpha=1 / 10$ & $44.34 \%$ & $43.89 \%$ & 0.102 \\
\hline Renyi $\alpha=1 / 9$ & $36.77 \%$ & $36.25 \%$ & 1.581 \\
\hline
\end{tabular}

Table II: Goodness of fit statistics model for data-2

\begin{tabular}{|c|c|c|c|}
\hline Data-3 & $\mathbf{R}^{2}$ & $R^{2}$ adj & MSE \\
\hline Classical & $38.48 \%$ & $37.98 \%$ & 9.452 \\
\hline Weighted with OLS & $52.52 \%$ & $52.13 \%$ & 0.952 \\
\hline Shannon & $44.29 \%$ & $43.84 \%$ & 3.566 \\
\hline Renyi $\alpha=0,5$ & $42.91 \%$ & $42.45 \%$ & 2.816 \\
\hline Renyi $\alpha=1,5$ & $43.39 \%$ & $42.93 \%$ & 3.085 \\
\hline Box-cox & $36.67 \%$ & $36.16 \%$ & 0.359 \\
\hline Tsallis $\alpha=1,5$ & $41.12 \%$ & $40.65 \%$ & 5.242 \\
\hline Tsallis $\alpha=0,5$ & $44.44 \%$ & $43.99 \%$ & 0.564 \\
\hline Tsallis $\alpha=1 / 9$ & $\mathbf{5 2 . 3} \%$ & $51.91 \%$ & 0.021 \\
\hline Tsallis $\alpha=1 / 10$ & $52.41 \%$ & $52.03 \%$ & 0.065 \\
\hline Renyi $\alpha=1 / 9$ & $40.6 \%$ & $40.12 \%$ & 1.435 \\
\hline
\end{tabular}

Table III: Goodness of fit statistics model for data-3

\begin{tabular}{|c|c|c|c|}
\hline Data-4 & $R^{2}$ & $R^{2}$ adj & MSE \\
\hline Classical & $56.47 \%$ & $56.27 \%$ & 8.150 \\
\hline Weighted with OLS & $61.55 \%$ & $61.38 \%$ & 0.925 \\
\hline Shannon & $60.11 \%$ & $59.93 \%$ & 3.180 \\
\hline Renyi $\alpha=0,5$ & $59.13 \%$ & $58.95 \%$ & 2.493 \\
\hline Renyi $\alpha=1,5$ & $59.46 \%$ & $59.28 \%$ & 2.737 \\
\hline Box-cox & $56.37 \%$ & $56.18 \%$ & 0.279 \\
\hline Tsallis $\alpha=1,5$ & $58.49 \%$ & $58.3 \%$ & 4.570 \\
\hline Tsallis $\alpha=0,5$ & $59.65 \%$ & $59.47 \%$ & 0.508 \\
\hline Tsallis $\alpha=1 / 9$ & $62.22 \%$ & $62.05 \%$ & 0.020 \\
\hline Tsallis $\alpha=1 / 10$ & $62.2 \%$ & $62.04 \%$ & 0.064 \\
\hline Renyi $\alpha=1 / 9$ & $57.67 \%$ & $57.48 \%$ & 1.25 \\
\hline
\end{tabular}

Table IV: Goodness of fit statistics model for data-3

\section{CONCLUSION}

The simplest model for regression analysis involves one independent variable and a constant. The variances of the equation may be equal for each level of independent variable or not. For equal variances the equation is called homoscedastic, otherwise the equation is called heteroscedastic. The heteroscedasticity could be detected visually when the data is shown in scatter diagram. On the other hand, for the numeric results White Test and Park's Test should be used to be more precise. In order to avoid from 
heteroscedasticity, generally weighting with error squares is one strategy. The Box-Cox transformation is also used to remedy. Alternatively, although it's not in common usage, weighting with reciprocal of entropy can be used, since entropy is a measure of uncertainty.In this study Shannon, Renyi and Tsallis entropies are used for determining the weights of The weihgted Least Squares (WLS) procedure. As can be seen on the tables above, introducing Tsallis and Rényi entropies in weighing may improve goodness of test statistics of a regression model like the coefficient of determination, the adjusted coefficient of determination, and the mean squares error.

\section{REFERENCES}

[1] RAWLINGS J.O.,PANTULA S.G., DICKEY D.A.(1998), “Applied Regression Analysis", Springer-Verlag,NY pp1-10.

[2] GUJARATI,D.M.(2004), "Basic Econometrics",The McGraw-Hill Companies fourth edition pp404,413.

[3] NETER,J.,WASSERMAN,W.,KUTNER,M.H.(1985), “Applied Linear Statistical Models", Richard D.Irwin, Inc. p263.

[4] UPTON,G.,COOK,I.(2006), Oxford Dictionary of Statistics,Second Edition, Oxford University Press.

[5] PIERCE,J.R. (1980)., "An Introduction to Information Theory Symbols, Signals and Noise", Dover Publications,Second, revised edition, NY, pp21-24

[6] KHINCHIN,A.I (1957)., " Mathematical Foundations of Information Theory", Dover Publications, NY, p7.

[7] COVER, T.M.; THOMAS, J.A.(2006), "Elements of Information Theory", Wiley Interscience (Second Edition), Hoboken, New Jersey, p45

[8] ASH, R.B.(1990), "Information Theory", Dover Publications, NY

[9] REZA,F.M. (1994)., "An Introduction to Information Theory", Dover Publications, NY.p268

[10] KULLBACK, S.(1996), "Information Theory and Statistics", Dover Publications, NY

[11] RENYI, A.(2007). "Probability Theory" ,Dover Publications, New York, $\mathrm{p} 23$.

[12] ZHANG, XING (2013). “Asymptotic Normality of Entropy Estimators", proposal submitted to the faculty of The University of North Carolina at Charlotte in partial fullfilment of the requirements for the degree of Doctor of Philosophy in Applied Mathematics, Charlotte )

[13] ULLAH, A.(1996), "Entropy, Divergence and Distance Measures with Econometric Applications, Journal of Statistical Planning and Inference", 49(1996), pp137-162

[14] PARDO, L.(2006), "Statistical Inference Based on Divergence Measures", Chapman\&HALL/CRC, pp1-34
APPENDIX:

Data-1
\begin{tabular}{|r|r|}
\hline$X$ & $Y$ \\
\hline 1,00 & 2,70 \\
\hline 1,00 & 1,72 \\
\hline 1,00 & 3,24 \\
\hline 1,00 & 4,28 \\
\hline 1,00 & 4,20 \\
\hline 2,00 & 6,44 \\
\hline 2,00 & 5,64 \\
\hline 2,00 & 6,26 \\
\hline 2,00 & 3,11 \\
\hline 2,00 & 5,13 \\
\hline 3,00 & 8,47 \\
\hline 3,00 & 5,35 \\
\hline 3,00 & 6,32 \\
\hline 3,00 & 4,31 \\
\hline 3,00 & 4,49 \\
\hline 4,00 & 3,89 \\
\hline 4,00 & 15,33 \\
\hline 4,00 & 12,65 \\
\hline 4,00 & 6,24 \\
\hline 4,00 & 9,16 \\
\hline 5,00 & 8,56 \\
\hline 5,00 & 11,61 \\
\hline 5,00 & 12,64 \\
\hline 5,00 & 17,32 \\
\hline 5,00 & 10,49 \\
\hline &
\end{tabular}

\begin{tabular}{|c|c|c|c|}
\hline \multicolumn{2}{|l|}{ Data-2 } & \multicolumn{2}{|l|}{ Data-3 } \\
\hline$X$ & $y$ & $x$ & \\
\hline 1,00 & 2,46 & 5,00 & 12,54 \\
\hline 1,00 & 4,12 & 5,00 & 17,02 \\
\hline 1,00 & 2,33 & 5,00 & 13,08 \\
\hline 1,00 & 1,81 & 5,00 & 6,20 \\
\hline 1,00 & 0,82 & 5,00 & 0,93 \\
\hline 1,00 & 3,53 & 5,00 & 6,76 \\
\hline 1,00 & 2,81 & 5,00 & 9,32 \\
\hline 1,00 & 3,37 & 5,00 & 11,55 \\
\hline 1,00 & 1,42 & 5,00 & 4,83 \\
\hline 1,00 & 2,60 & 5,00 & 6,53 \\
\hline 1,00 & 2,28 & 5,00 & 9,75 \\
\hline 1,00 & 4,04 & 5,00 & 14,97 \\
\hline 1,00 & 4,93 & 5,00 & 0,70 \\
\hline 1,00 & 3,48 & 5,00 & 6,86 \\
\hline 1,00 & 4,27 & 5,00 & 13,65 \\
\hline 1,00 & 4,59 & 5,00 & 10,37 \\
\hline 1,00 & 1,44 & 5,00 & 15,28 \\
\hline 1,00 & 3,09 & 5,00 & 10,70 \\
\hline 1,00 & 2,65 & 5,00 & 6,13 \\
\hline 1,00 & 2,73 & 5,00 & 14,25 \\
\hline 1,00 & 2,78 & 5,00 & 8,41 \\
\hline 1,00 & 3,28 & 5,00 & 12,01 \\
\hline 1,00 & 1,09 & 5,00 & 16,71 \\
\hline 1,00 & 4,72 & 5,00 & 5,06 \\
\hline 1,00 & 2,91 & 5,00 & 10,96 \\
\hline 2,00 & 2,73 & 4,00 & 6,42 \\
\hline 2,00 & 6,75 & 4,00 & 5,88 \\
\hline 2,00 & 8,11 & 4,00 & 10,80 \\
\hline 2,00 & 5,29 & 4,00 & 12,96 \\
\hline 2,00 & 5,32 & 4,00 & 10,78 \\
\hline 2,00 & 6,59 & 4,00 & 14,48 \\
\hline 2,00 & 4,84 & 4,00 & 6,78 \\
\hline 2,00 & 5,91 & 4,00 & 7,67 \\
\hline 2,00 & 7,24 & 4,00 & 8,61 \\
\hline 2,00 & 5,57 & 4,00 & 13,07 \\
\hline 2,00 & 5,47 & 4,00 & 6,38 \\
\hline 2,00 & 9,15 & 4,00 & 5,79 \\
\hline 2,00 & 4,78 & 4,00 & 10,36 \\
\hline 2,00 & 6,16 & 4,00 & 8,67 \\
\hline 2,00 & 5,19 & 4,00 & 6,79 \\
\hline 2,00 & 6,23 & 4,00 & 8,74 \\
\hline 2,00 & 3,77 & 4,00 & 9,80 \\
\hline 2,00 & 5,61 & 4,00 & 15,02 \\
\hline 2,00 & 3,38 & 4,00 & 8,49 \\
\hline 2,00 & 4,48 & 4,00 & 14,33 \\
\hline 2,00 & 6,94 & 4,00 & 12,14 \\
\hline 2,00 & 4,59 & 4,00 & 3,63 \\
\hline 2,00 & 6,48 & 4,00 & 12,36 \\
\hline 2,00 & 5,27 & 4,00 & 1,04 \\
\hline 2,00 & 6,20 & 4,00 & 3,49 \\
\hline 3,00 & 6,57 & 3,00 & 7,04 \\
\hline 3,00 & 7,42 & 3,00 & 5,88 \\
\hline 3,00 & 5,77 & 3,00 & 3,78 \\
\hline 3,00 & 5,34 & 3,00 & 9,80 \\
\hline 3,00 & \begin{tabular}{|r}
15,82 \\
\end{tabular} & 3,00 & 14,16 \\
\hline 3,00 & 5,27 & 3,00 & 5,37 \\
\hline 3,00 & 6,80 & 3,00 & 3,55 \\
\hline 3,00 & 6,79 & 3,00 & 6,94 \\
\hline 3,00 & 4,05 & 3,00 & 2,82 \\
\hline 3,00 & 5,57 & 3,00 & 10,93 \\
\hline 3,00 & 9,65 & 3,00 & 4,06 \\
\hline 3,00 & 2,14 & 3,00 & 4,20 \\
\hline 3,00 & \begin{tabular}{|l}
10,26 \\
\end{tabular} & 3,00 & 5,93 \\
\hline
\end{tabular}

Data-4

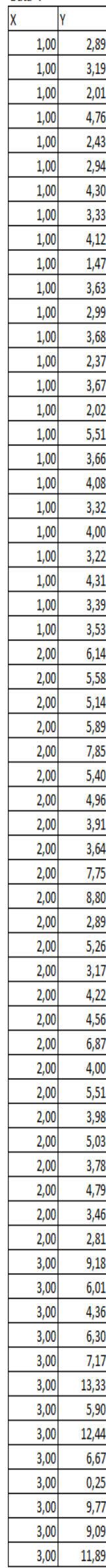


International Journal of Engineering and Applied Sciences (IJEAS)

ISSN: 2394-3661, Volume-6, Issue-5, May 2019

\begin{tabular}{|c|c|}
\hline 3,00 & 4,60 \\
\hline 3,00 & 4,54 \\
\hline 3,00 & 6,67 \\
\hline 3,00 & 6,22 \\
\hline 3,00 & 9,93 \\
\hline 3,00 & 4,75 \\
\hline 3,00 & 12,13 \\
\hline 3,00 & 7,00 \\
\hline 3,00 & 10,10 \\
\hline 3,00 & 10,09 \\
\hline 3,00 & 4,93 \\
\hline 3,00 & 8,98 \\
\hline 4,00 & 16,57 \\
\hline 4,00 & 5,55 \\
\hline 4,00 & 10,46 \\
\hline 4,00 & 3,20 \\
\hline 4,00 & 9,67 \\
\hline 4,00 & 13,77 \\
\hline 4,00 & 9,78 \\
\hline 4,00 & 15,98 \\
\hline 4,00 & 11,30 \\
\hline 4,00 & 7,43 \\
\hline 4,00 & 7,98 \\
\hline 4,00 & 16,64 \\
\hline 4,00 & 14,49 \\
\hline 4,00 & 2,52 \\
\hline 4,00 & 7,78 \\
\hline 4,00 & 11,71 \\
\hline 4,00 & 7,55 \\
\hline 4,00 & 9,37 \\
\hline 4,00 & 11,13 \\
\hline 4,00 & 2,54 \\
\hline 4,00 & 10,30 \\
\hline 4,00 & 0,63 \\
\hline 4,00 & 6,40 \\
\hline 4,00 & 9,48 \\
\hline 4,00 & 8,12 \\
\hline 5,00 & 13,91 \\
\hline 5,00 & 2,71 \\
\hline 5,00 & 6,88 \\
\hline 5,00 & 13,89 \\
\hline 5,00 & 10,88 \\
\hline 5,00 & 19,69 \\
\hline 5,00 & 11,45 \\
\hline 5,00 & 9,15 \\
\hline 5,00 & 7,55 \\
\hline 5,00 & 1,31 \\
\hline 5,00 & 9,05 \\
\hline 5,00 & 16,04 \\
\hline 5,00 & 4,45 \\
\hline 5,00 & 7,99 \\
\hline 5,00 & 11,89 \\
\hline 5,00 & 8,72 \\
\hline 5,00 & 10,34 \\
\hline 5,00 & 10,67 \\
\hline 5,00 & 13,68 \\
\hline 5,00 & 4,35 \\
\hline 5,00 & 6,29 \\
\hline 5,00 & 7,77 \\
\hline 5,00 & 8,57 \\
\hline 5,00 & 15,31 \\
\hline 5,00 & 4,07 \\
\hline
\end{tabular}

\begin{tabular}{|c|c|}
\hline 3,00 & 10,73 \\
\hline 3,00 & 5,27 \\
\hline 3,00 & 10,85 \\
\hline 3,00 & 12,08 \\
\hline 3,00 & 9,29 \\
\hline 3,00 & 8,75 \\
\hline 3,00 & 2,28 \\
\hline 3,00 & 10,30 \\
\hline 3,00 & 7,22 \\
\hline 3,00 & 3,82 \\
\hline 3,00 & 6,10 \\
\hline 3,00 & 4,10 \\
\hline 2,00 & 5,22 \\
\hline 2,00 & 8,32 \\
\hline 2,00 & 4,32 \\
\hline 2,00 & 5,57 \\
\hline 2,00 & 5,95 \\
\hline 2,00 & 6,12 \\
\hline 2,00 & 4,72 \\
\hline 2,00 & 5,78 \\
\hline 2,00 & 6,20 \\
\hline 2,00 & 3,43 \\
\hline 2,00 & 6,06 \\
\hline 2,00 & 5,37 \\
\hline 2,00 & 7,18 \\
\hline 2,00 & 2,50 \\
\hline 2,00 & 2,22 \\
\hline 2,00 & 4,71 \\
\hline 2,00 & 6,39 \\
\hline 2,00 & 4,26 \\
\hline 2,00 & 3,73 \\
\hline 2,00 & 7,95 \\
\hline 2,00 & 6,43 \\
\hline 2,00 & 3,67 \\
\hline 2,00 & 6,86 \\
\hline 2,00 & 4,71 \\
\hline 2,00 & 6,15 \\
\hline 1,00 & 3,09 \\
\hline 1,00 & 2,03 \\
\hline 1,00 & 3,40 \\
\hline 1,00 & 3,02 \\
\hline 1,00 & 1,49 \\
\hline 1,00 & 2,42 \\
\hline 1,00 & 3,63 \\
\hline 1,00 & 2,51 \\
\hline 1,00 & 3,10 \\
\hline 1,00 & 2,11 \\
\hline 1,00 & 1,77 \\
\hline 1,00 & 3,39 \\
\hline 1,00 & 4,41 \\
\hline 1,00 & 2,94 \\
\hline 1,00 & 1,61 \\
\hline 1,00 & 4,07 \\
\hline 1,00 & 6,07 \\
\hline 1,00 & 4,45 \\
\hline 1,00 & 2,53 \\
\hline 1,00 & 2,83 \\
\hline 1,00 & 2,40 \\
\hline 1,00 & 3,08 \\
\hline 1,00 & 4,17 \\
\hline 1,00 & 3,19 \\
\hline 1,00 & 3,01 \\
\hline
\end{tabular}

\begin{tabular}{|c|c|}
\hline 3,00 & 7,82 \\
\hline 3,00 & 9,22 \\
\hline 3,00 & 9,99 \\
\hline 3,00 & 7,10 \\
\hline 3,00 & 8,43 \\
\hline 3,00 & 7,79 \\
\hline 3,00 & 2,94 \\
\hline 3,00 & 9,37 \\
\hline 3,00 & 2,63 \\
\hline 3,00 & 2,83 \\
\hline 3,00 & 10,87 \\
\hline 3,00 & 3,38 \\
\hline 4,00 & 5,08 \\
\hline 4,00 & 16,01 \\
\hline 4,00 & 8,05 \\
\hline 4,00 & 16,92 \\
\hline 4,00 & 10,13 \\
\hline 4,00 & 16,23 \\
\hline 4,00 & 11,79 \\
\hline 4,00 & 8,35 \\
\hline 4,00 & 13,84 \\
\hline 4,00 & 10,11 \\
\hline 4,00 & 4,20 \\
\hline 4,00 & 7,71 \\
\hline 4,00 & 7,85 \\
\hline 4,00 & 4,51 \\
\hline 4,00 & 9,97 \\
\hline 4,00 & 8,61 \\
\hline 4,00 & 9,37 \\
\hline 4,00 & 8,32 \\
\hline 4,00 & 8,48 \\
\hline 4,00 & 9,73 \\
\hline 4,00 & 7,49 \\
\hline 4,00 & 2,86 \\
\hline 4,00 & 11,70 \\
\hline 4,00 & 12,75 \\
\hline 4,00 & 14,98 \\
\hline 5,00 & 20,01 \\
\hline 5,00 & 15,44 \\
\hline 5,00 & 11,93 \\
\hline 5,00 & 13,00 \\
\hline 5,00 & 20,57 \\
\hline 5,00 & 16,85 \\
\hline 5,00 & 10,39 \\
\hline 5,00 & 14,46 \\
\hline 5,00 & 12,03 \\
\hline 5,00 & 22,22 \\
\hline 5,00 & 8,63 \\
\hline 5,00 & 11,92 \\
\hline 5,00 & 17,18 \\
\hline 5,00 & 13,80 \\
\hline 5,00 & 8,91 \\
\hline 5,00 & 10,50 \\
\hline 5,00 & 15,52 \\
\hline 5,00 & 17,63 \\
\hline 5,00 & 12,94 \\
\hline 5,00 & 9.44 \\
\hline 5,00 & 15,61 \\
\hline 5,00 & 12,69 \\
\hline 5,00 & 7,3 \\
\hline 5,00 & 10,28 \\
\hline 5,00 & 17,79 \\
\hline 4,00 & 4,9 \\
\hline 4,00 & 6, \\
\hline 4,00 & 10, \\
\hline
\end{tabular}

\begin{tabular}{|c|c|c|c|}
\hline 4,00 & 9,99 & & \\
\hline 4,00 & 10,24 & & \\
\hline 4,00 & 2,33 & & \\
\hline 4,00 & 8,46 & & \\
\hline 4,00 & 5,78 & & \\
\hline 4,00 & 9,65 & & \\
\hline 4,00 & 8,91 & & \\
\hline 4,00 & 8,72 & & \\
\hline 4,00 & 9,74 & & \\
\hline 4,00 & 10,55 & & \\
\hline 4,00 & 5,95 & & \\
\hline 4,00 & 11,66 & & \\
\hline 4,00 & 19,10 & & \\
\hline 4,00 & 10,84 & & \\
\hline 4,00 & 5,62 & & \\
\hline 4,00 & 13,66 & & \\
\hline 4,00 & 11,47 & & \\
\hline 4,00 & 4,43 & & \\
\hline 4,00 & 11,06 & & \\
\hline 4,00 & 7,59 & & \\
\hline 4,00 & 12,73 & & \\
\hline 4,00 & 12,45 & & \\
\hline 3,00 & 4,09 & & \\
\hline 3,00 & 7,14 & & \\
\hline 3,00 & 6,80 & & \\
\hline 3,00 & 1,64 & & \\
\hline 3,00 & 4,28 & & \\
\hline 3,00 & 6,22 & & \\
\hline 3,00 & 9,65 & & \\
\hline 3,00 & 9,99 & & \\
\hline 3,00 & 2,82 & & \\
\hline 3,00 & 6,72 & & \\
\hline 3,00 & 4,77 & & \\
\hline 3,00 & 1,22 & 2,00 & 2,55 \\
\hline 3,00 & 6,12 & 2,00 & 3,84 \\
\hline 3,00 & 6,59 & 2,00 & 4,07 \\
\hline 3,00 & 4,21 & 2,00 & 7,17 \\
\hline 3,00 & 9,62 & 2,00 & 6,89 \\
\hline 3,00 & 4,91 & 2,00 & 8,83 \\
\hline 3,00 & 0,92 & 2,00 & 4,88 \\
\hline 3,00 & 8,66 & 1,00 & 2,70 \\
\hline 3,00 & 5,04 & 1,00 & 3,53 \\
\hline 3,00 & 4,85 & 1,00 & 3,92 \\
\hline 3,00 & 7,53 & 1,00 & 3,26 \\
\hline 3,00 & 10,18 & 1,00 & 2,23 \\
\hline 3,00 & 5,58 & 1,00 & 3,90 \\
\hline 3,00 & 11,98 & 1,00 & 2,10 \\
\hline 2,00 & 6,63 & 1,00 & 3,32 \\
\hline 2,00 & 6,35 & 1,00 & 1,73 \\
\hline 2,00 & 0,67 & 1,00 & 2,87 \\
\hline 2,00 & 5,02 & 1,00 & 1,64 \\
\hline 2,00 & 5,58 & 1,00 & 3,50 \\
\hline 2,00 & 6,51 & 1,00 & 2,09 \\
\hline 2,00 & 5,81 & 1,00 & 3,45 \\
\hline 2,00 & 7,37 & 1,00 & 2,01 \\
\hline 2,00 & 3,33 & 1,00 & 3,72 \\
\hline 2,00 & 8,07 & 1,00 & 2,85 \\
\hline 2,00 & 2,50 & 1,00 & 2,80 \\
\hline 2,00 & 5,46 & 1,00 & 3,11 \\
\hline 2,00 & 4,11 & 1,00 & 2,11 \\
\hline 2,00 & 5,24 & 1,00 & 1,56 \\
\hline 2,00 & 1,62 & 1,00 & 4,23 \\
\hline 2,00 & 3,47 & 1,00 & 3,56 \\
\hline 2,00 & 6,54 & 1,00 & 3,87 \\
\hline 2,00 & 2,10 & 1,00 & 2,35 \\
\hline
\end{tabular}

Table V:Data 